\title{
Spinal Injury
}

National Cancer Institute

\section{Source}

National Cancer Institute. Spinal Injury. NCI Thesaurus. Code C50751.

Damage to the spine that results in impaired function. 